\title{
PROJECTIVITIES WITH FIXED POINTS ON EVERY LINE OF THE PLANE
}

\author{
REINHOLD BAER
}

The system of fixed elements of a projectivity contains with any two points the line connecting them and with any two lines their intersection. It is, therefore, in its structure very much like a subplane of the plane under consideration; and thus one may expect the structure of the projectivity to be dominated by the structure of the system of its fixed elements, provided this system is not "too small." To substantiate this we propose to investigate in this note a class of projectivities which we term quasi-perspectivities. They are characterized by the property that every line carries a fixed point or, equivalently, that every point is on some fixed line.

Every perspectivity is a quasi-perspectivity, and a quasi-perspectivity is not a perspectivity if, and only if, the system of its fixed elements is a projective subplane. Involutions are quasi-perspectivities too, and if the Theorem of Pappus is valid in the plane under consideration, then every quasi-perspectivity is a perspectivity or an involution. But already in the projective plane over the field of real quaternions there exist quasi-perspectivities which are neither perspectivities nor involutions, and we give a complete survey of the quasi-perspectivities in Desarguesian projective planes.

Our results become particularly striking in the case of finite projective planes. If every line in such a plane carries $n+1$ points, then we may show that there do not exist projectivities possessing exactly $n$ fixed points, that a projectivity is a quasi-perspectivity if, and only if, the number of its fixed points is at least $n+1$, and that it is a perspectivity if, and only if, the number of its fixed points is $n+1$ or $n+2$. If a quasi-perspectivity is not a perspectivity, then $n=i^{2}$ where $i+1$ is the number of fixed points on a fixed line.

The following notations will be used throughout.

We consider a projective plane $\Pi$ in which the Theorem of Desargues may or may not hold. ${ }^{1}$ If $P$ and $Q$ are two different points in $\Pi$, then $P+Q$ is the uniquely determined line passing through $P$ and $Q$; if $h$ and $k$ are two different lines, then $h k$ is the uniquely determined point in which they meet.

A projectivity $\phi$ is a 1:1 and exhaustive correspondence between the

Presented to the Society, April 27, 1946; received by the editors October 18, 1945.

1 For a definition of "projective plane" see, for example, Baer [1, p. 138]. Numbers in brackets refer to the Bibliography at the end of the paper. 
points of $\Pi$ and between the lines of $\Pi$ such that the point $P$ is on the line $h$ if, and only if, the image point $P \phi$ is on the image line $h \phi$.

The point $P$ is a fixed point of the projectivity $\phi$ if $P=P \phi$, and fixed lines are defined likewise. The system of all the fixed elements of $\phi$ (fixed points as well as fixed lines) shall be denoted by $\Phi(\phi)$.

THEOREM 1. The following properties of a projectivity $\phi$ imply each other.

(i) $P, P \phi$ and $P \phi^{2}$ are collinear for every point $P$.

(ii) Every point lies on a fixed line.

(iii) Every line carries a fixed point.

(iv) $h, h \phi$ and $h \phi^{2}$ are copunctual for every line $h$.

Proof. If (i) is true and $P$ a point, then we distinguish two cases.

Case 1. $P$ is not a fixed point. Then $P \neq P \phi$ and $h=P+P \phi$ is a well determined line containing $P \phi^{2}$. But $h \phi=P \phi+P \phi^{2}=h$ shows that the fixed line $h$ passes through the point $P$.

Case 2. $P$ is a fixed point. There exists a point $Q \neq P$. If $Q$ is a fixed point, then $P+Q$ is the desired fixed line passing through $P$. Thus we assume that $Q$ is not a fixed point. Then it follows as under Case 1 that the line $q=Q+Q \phi$ is a fixed line. If $P$ is on $q$, then $q$ is the desired fixed line through $P$. Hence we assume that $P$ is not on $q$. There exists a point $R \neq P$ which is not on $q$. If $R$ is a fixed point, then $P+R$ is a fixed line through $P$. Thus we assume that $R$ is not a fixed point. Then we show as before that $R+R \phi=r$ is a fixed line. But $r \neq q$, since $R$ is not on $q$. Thus $r$ and $q$ meet in a fixed point $S$. This fixed point $S$ is different from $P$, since $P$ is not on $q$, but $S$ is on $q$. Then $P+S$ is a fixed line through $P$. Hence we have shown in every case that (i) implies (ii).

Assume now the validity of (ii). If $P$ is some point, then there exists a fixed line $p$, passing through $P$. Since $P$ is on $p, P \phi^{i}$ is on $p \phi^{i}=p$ so that $P, P \phi$ and $P \phi^{2}$ are collinear as points on the same line $p$. Thus (i) is a consequence of (ii); and we have shown the equivalence of (i) and (ii).

By duality we have the equivalence of (iv) and (iii).

Assume now the validity of (ii) and consider a line $h$. If $h$ is a fixed line, then consider a point $P$ not on $h$. This point $P$ lies by (ii) on a fixed line $p$ which is different from $h$. Hence $p$ and $h$ are two different fixed lines meeting in a fixed point on $h$. Assume next that $h$ is not a fixed line. Then $h$ and $h \phi$ meet in a point $K$ and this point $K$ lies by (ii) on a fixed line $k$. Clearly $K=h k$, since $h$ and $k$ are different, the second line being fixed and the first one not. Hence $K \phi=(h \phi)(k \phi)$ $=(h \phi) k=K$, showing that $h$ carries the fixed point $K$. Thus we proved 
that (iii) is a consequence of (ii); and it follows by duality that (ii) is a consequence of (iii), completing the proof.

Every projectivity $\phi \neq 1$ meeting the requirements (i) to (iv) of Theorem 1 shall be termed a quasi-perspectivity. It is clear that every perspectivity meets requirement (ii), since a perspectivity possesses a line all of whose points are fixed points, ${ }^{2}$ and since every line meets this line in a fixed point. But every involution meets requirement (i) because of $P=P \phi^{2}$, showing that perspectivities as well as involutions are quasi-perspectivities.

COROLlARY 1. If $\phi$ is a quasi-perspectivity, and if the line $h$ is not a fixed line, then $h$ carries one and only one fixed point.

We infer from Theorem 1, (iii), that $h$ carries fixed points. But $h$ would be a fixed line, if $h$ carried two fixed points.

COROLlary 2. (a) The quasi-perspectivity $\phi$ possesses fixed lines carrying but one fixed point if, and only if, $\phi$ is an elation. ${ }^{3}$

(b) If the quasi-perspectivity $\phi$ is not an elation, then a necessary and sufficient condition for the line $h$ to be a fixed line is the existence of at least two different fixed points on $h$.

Proof. If $\phi$ is an elation, the line $h$ its axis and the point $H$ on $h$ its center, then a point is a fixed point if, and only if, it is on $h$, and a line is a fixed line if, and only if, it passes through $H$. Clearly the lines through $H$ which are different from $h$ are fixed lines carrying one and only one fixed point, namely $H$.

Suppose conversely that $\phi$ is a quasi-perspectivity and that the fixed line $h$ of $\phi$ carries one and only one fixed point $H$. If $k$ is a fixed line different from $h$, then $k$ and $h$ meet in a fixed point on $h$ so that $k$ passes through $H$ as the only fixed point on $h$. If $P$ is a point not on $h$, then either $P$ is a fixed point and $P+H$ is a fixed line; or else $P$ is not a fixed point and $P+P \phi$ is by Theorem 1, (i), a fixed line which necessarily passes through $H$. Thus $P+H$ is always a fixed line. Hence every line through $H$ is a fixed line so that $H$ is a center of $\phi$. But projectivities possess an axis whenever they possess a center, ${ }^{4}$ and the axis is a fixed line so that it must pass through the center $H$. Consequently $\phi$ is an elation, completing the proof of (a). (b) is an immediate consequence of (a) and Corollary 1.

2 Namely the axis of the perspectivity; see Veblen-Young [1, p. 72].

3 An elation is a perspectivity whose center is on its axis; see Veblen-Young [1, p. 72].

4 See, for example, Baer [1, Corollary 2.3, p. 140]. 
Preparatory to characterizing the perspectivities among the quasiperspectivities we introduce the following concept. The set $\Gamma$ of points and lines is a closed configuration ${ }^{5}$ if it meets the following requirements:

If $P \neq Q$ are points in $\Gamma$, then $P+Q$ is in $\Gamma$. If $h \neq k$ are lines in $\Gamma$, then $h k$ is in $\Gamma$.

We note that the set of fixed elements of a projectivity is always a closed configuration. Another example are the projective subplanes ${ }^{6}$ of $\Pi$.

Lemma $1 .^{7}$ The closed configuration $\Gamma$ which contains both points and lines is not a projective subplane if, and only if, there exist a point $H$ and a line $h$ with the following properties: every point in $\Gamma$ with the possible exception of $H$ is on $h$; and every line in $\Gamma$ with the possible exception of $h$ passes through $H$.

Proof. The sufficiency of this condition is obvious. Assume conversely that $\Gamma$ is not a projective subplane. Then there exists a line $w$ in $\Gamma$ carrying at most two points in $\Gamma$.

Case 1. No point in $\Gamma$ is on $w$. Then $w$ is the only line in $\Gamma$, since the existence of a line $v \neq w$ in $\Gamma$ would imply that the intersection of $v$ and $w$ would be on $w$ and in $\Gamma$. There exists at least one point $W$ in $\Gamma$. If there would exist a point $V \neq W$ in $\Gamma$, then $V+W \neq w$, since neither $V$ nor $W$ is on $w$; but the line $V+W$ would be in $\Gamma$ which has been shown to be impossible. Thus $\Gamma$ consists of the line $w$ and the point $W$ not on w.

Case 2. There is one and only one point $W$ in $\Gamma$ and on $w$. If $x$ is a line in $\Gamma, x \neq w$, then $x$ and $w$ meet in a point which is both in $\Gamma$ and on $w$. Hence $x$ passes through $W$, showing that every line in $\Gamma$ passes through $W$.

If $P \neq Q$ are points in $\Gamma$, then $P+Q$ is a line in $\Gamma$ so that $P+Q$ passes through $W$. This implies the collinearity of all the points in $\Gamma$, proving again the validity of our condition.

Case 3. There exist exactly two points in $\Gamma$ which are on $w$. Suppose $W^{\prime}$ and $W^{\prime \prime}$ are the two different points in $\Gamma$ which are on $w$. If $x$ is any line in $\Gamma, x \neq w$, then $x$ and $w$ meet in a point in $\Gamma$ and on $w$. Hence $x$ passes through one and only one of the two points $W^{\prime}$ and $W^{\prime \prime}$.

5 The term "configuration" refers usually only to finite systems, a restriction that we do not impose on this concept. M. Hall [1] terms "degenerate projective plane" what we would call a closed configuration which is not a subplane.

- These are sometimes referred to as "nets."

${ }^{7}$ This lemma has been stated without proof by M. Hall [1, p. 232]. 
Our condition is certainly satisfied by $\Gamma$ if there exists at most one point in $\Gamma$ which is not on $w$. Thus let us assume the existence of two different points $Q^{\prime}, Q^{\prime \prime}$ in $\Gamma$ which are not on $w$. Then $Q^{\prime}+Q^{\prime \prime}$ belongs to $\Gamma$, is not $w$, and passes therefore through one and only one of the points $W^{\prime}$ and $W^{\prime \prime}$. We assume without loss in generality that $W^{\prime}, Q^{\prime}$ and $Q^{\prime \prime}$ are collinear.

Suppose now that the point $U$ in $\Gamma$ is neither on $w$ nor on $Q^{\prime}+Q^{\prime \prime}$. Then $Q^{\prime}+U$ is a line in $\Gamma$ which cannot pass through $W^{\prime}$, since otherwise $U$ would be on $Q^{\prime}+W^{\prime}=W^{\prime}+W^{\prime \prime}$. Thus $Q^{\prime}+U$ passes through $W^{\prime \prime}$; likewise we see that $Q^{\prime \prime}+U$ passes through $W^{\prime \prime}$. If the lines $Q^{\prime}+U$ and $Q^{\prime \prime}+U$ were different, then they would meet in $U=W^{\prime \prime}$ which is impossible, and if the lines $Q^{\prime}+U$ and $Q^{\prime \prime}+U$ were equal, then $U$ would be on $Q^{\prime}+Q^{\prime \prime}$ which we excluded too. It follows that the only point not on $Q^{\prime}+Q^{\prime \prime}$, but in $\Gamma$, is $W^{\prime \prime}$, and $w$ is readily seen to be the only line in $\Gamma$ which does not pass through $W^{\prime \prime}$. Thus our condition is satisfied, completing the proof of Lemma 1.

THEOREM 2. The quasi-perspectivity $\phi$ is a perspectivity if, and only if, the system $\Phi$ of fixed elements of $\phi$ is not a projective subplane.

Proof. If $\phi$ is a perspectivity, then there exists a point $H$ and a line $h$ such that the set of fixed points, with the possible exception of $H$, is just the set of points on $h$, and such that the set of fixed lines, with the possible exception of $h$, is just the pencil of lines through $H$. It is clear that $\Phi$ is not a projective subplane.

Assume conversely that $\Phi$ is not a projective subplane. There exist fixed points and fixed lines, since by Theorem 1 every point is on a fixed line and every line passes through a fixed point. We note furthermore that $\Phi$ is a closed configuration. Hence we may infer from Lemma 1 the existence of a fixed point $H$ and of a fixed line $h$ with the following properties:

If $P$ is a fixed point, $P \neq H$, then $P$ is on $h$; and if $k$ is a fixed line, $k \neq h$, then $k$ passes through $H$.

Suppose that $Q$ is a point on $h$. There exists a line $q \neq h$ through $Q$ which does not pass $H$. This line $q$ carries a fixed point which is different from $H$ and which therefore is on $h$. But $q$ and $h$ meet in $Q$, proving that $Q$ is a fixed point. Thus every point on $h$ is a fixed point; that every line through $H$ is a fixed line is shown likewise. The point $H$ is the center and the line $h$ is the axis of $\phi$, proving that $\phi$ is a perspectivity.

A closed configuration $\Gamma$ in the projective plane $\Pi$ is termed maxi$m a l$ if it is different from $\Pi$, and if $\Pi$ is the only closed configuration which contains $\Gamma$ as a proper subset. One deduces without difficulty 
from Lemma 1 that the maximal closed configurations which are not projective subplanes consist of a line $h$, a point $H$, the points on $h$ and the lines through $H$.

THEOREM 3. The systems of fixed elements of quasi-perspectivities are maximal closed configurations.

Proof. Suppose that $\Phi$ is the system of fixed elements of the quasiperspectivity $\phi$. Then $\Phi$ is a closed configuration. If $\Phi$ is a proper subset of the closed configuration $\Gamma$, then we may assume without loss in generality that $\Gamma$ contains a point $P$ which is not in $\Phi$. Every line through $P$ carries a fixed point of $\phi$ which is necessarily different from $P$. Hence every line through $P$ is in $\Gamma$. If $h$ is a fixed line not passing through $P$, then every point on $h$ is the intersection of $h$ and of a line through $P$ which both belong to $\Gamma$. Hence every point on $h$ is in $\Gamma$. Every point is on a fixed line (Theorem 1), and thus every point is in $\Gamma$ with the possible exception of the points on the uniquely determined fixed line through $P$ (Corollary 1 ). Now it is easily seen that every line and, therefore, every point is in $\Gamma$, completino the proof.

It is very easy to construct examples of maximal closed configurations which are not the systems of fixed elements of a suitable projectivity and examples of maximal closed configurations which are the systems of fixed elements of a suitable projectivity, but not of a quasiperspectivity.

If $\Gamma$ is a maximal closed configuration, then we may form the group $A=A(\Gamma)$ of all the projectivities which leave invariant every element in $\Gamma$. It is possible that $A=1$. The following facts are immediate consequences of Theorem 1 and the fact that the systems of fixed elements are closed configurations.

Corollary 3. Suppose that $\Gamma$ is a maximal closed configuration in the projective plane $\Pi$, that every point in $\Pi$ is on a line in $\Gamma$, and that every line in $\Pi$ passes through a point in $\Gamma$.

(a) If $\phi \neq 1$ is in $\mathrm{A}(\Gamma)$, then $\phi$ is a quasi-perspectivity whose system of fixed elements is $\Gamma$.

(b) If $\phi$ and $\gamma$ are in $\mathrm{A}(\Gamma)$, and if there exists an element not in $\Gamma$ on which $\phi$ and $\gamma$ have the same effect, then $\phi=\gamma$.

If the point $P$ is not a point on $\Gamma$, and if $P^{*}$ is the set of all the images of $P$ under projectivities in $\mathrm{A}(\Gamma)$, then it follows from the preceding Corollary 3 that the points in $P^{*}$ are collinear and that $A(\Gamma)$ induces a regular group of permutations in $P^{*}$. If in particular $A(\Gamma)$ is a finite group, then all systems $P^{*}$, for $P$ not in $\Gamma$, contain the same number of elements, this number being the order of the group $A(\Gamma)$. 
The following lemma will be convenient for the construction of projectivities whose system of fixed elements is a subplane.

Lemma 2. The system $\Phi$ of fixed elements of the projectivity $\phi$ is a projective subplane of the projective plane II if, and only if, there exist four fixed points of $\phi$ no three of which are collinear. ${ }^{8}$

Proof. The necessity of the condition being obvious, let us assume the existence of four fixed points $U, V, W$ and $Z$ of $\phi$ such that no three of these four points are collinear. If $P$ is any fixed point of $\phi$, then we have to show that three different fixed lines pass through $P$. If $P$ happens to be one of the four basic points, say $P=U$, then $U+V, U+W$ and $U+Z$ are three different fixed lines passing through $P$. If $P$ is different from the four basic points, then the lines $P+U$, $P+V, P+W$ and $P+Z$ are fixed lines passing through $P$. Thus we have proved our contention, provided three of these lines are different. Assume therefore that only two of these lines are different. Noting the impossibility that three of these lines are equal, we may assume without loss in generality that $P+U=P+V$ and $P+W=P+Z$ so that $P$ is the intersection of the fixed lines $U+V$ and $W+Z$. But the lines $W+U$ and $V+Z$ meet in a fixed point $M$ and the line $M+P$ is the desired third fixed line through $P$.

Of the four fixed lines $U+V, V+W, W+Z$ and $Z+U$ no three are copunctual. Hence we infer by duality from the result of the first paragraph that there are on every fixed line of $\phi$ at least three fixed points; and this completes the proof of the lemma, since fixed lines meet in fixed points and since the lines through fixed points are fixed lines.

Lemma 3. If $\Pi$ is the projective plane over the (not necessarily commutative) field ${ }^{9} G$, and if $\phi \neq 1$ is a projectivity of $\Pi$, then the following condition is necessary and sufficient for the system $\Phi$ of fixed elements of $\phi$ to be a projective subplane of $\Pi$ :

$\left.{ }^{*}\right)$ There exists an automorphism $\alpha \neq 1$ of the field $G$ and a system of homogeneous coordinates of $\Pi$ such that the point with coordinates $\left(x_{0}, x_{1}, x_{2}\right)$ is mapped by $\phi$ upon the point with coordinates $\left(x_{0}{ }^{\alpha}, x_{1}^{\alpha}, x_{2}^{\alpha}\right)$.

This may be deduced by the customary arguments ${ }^{10}$ from Lemma 2.

8 M. Hall [1, p. 231] uses this property as a postulate instead of the equivalent requirements that every line carries at least three points and every point is on at least three lines on which requirement our discussion is based; see Baer [1, p. 138].

- The word "field" shall always be used in this general sense.

${ }^{10} \mathrm{See}$, for example, Baer [2] or Brauer [1]. 
Lemma 4. The projectivity $\phi$ of the projective plane II over the field $G$ which is determined by the automorphism $\alpha$ of $G$ according to Lemma 3, $\left(^{*}\right)$, is a quasi-perspectivity if, and only if, there exists a number $c \neq 0$ in $G$ such that $c^{\alpha}=c$ and $d^{11}\left(x^{\alpha}-x\right)^{\alpha}=c\left(x^{\alpha}-x\right)$ for every $x$ in $G$.

PROoF. If the condition is satisfied, then

$$
\left(x_{0}^{\alpha^{2}}, x_{1}^{\alpha^{2}}, x_{2}^{\alpha^{2}}\right)=(1+c)\left(x_{0}^{\alpha}, x_{1}^{\alpha}, x_{2}^{\alpha}\right)-c\left(x_{0}, x_{1}, x_{2}\right) ;
$$

and we infer from Theorem 1, (i), that $\phi$ is a quasi-perspectivity.

Assume conversely that $\phi$ is a quasi-perspectivity. Consider the point with coordinates $(1, x, y)$ for $x$ and $y$ in $G$. Then we infer from Theorem 1, (i), the existence of numbers $r, s$ in $G$ such that $\left(1, x^{\alpha^{2}}, y^{\alpha^{2}}\right)=r\left(1, x^{\alpha}, y^{\alpha}\right)+s(1, x, y)$ or $1=r+s, x^{\alpha^{2}}=r x^{\alpha}+s x$, $y^{\alpha^{2}}=r y^{\alpha}+s y$; this is equivalent to the following statement:

If $x$ and $y$ are numbers in $G$, then there exists a number $s$ in $G$ such that $x^{\alpha^{2}}=x^{\alpha}+s\left(x-x^{\alpha}\right)$ and $y^{\alpha^{2}}=y^{\alpha}+s\left(y-y^{\alpha}\right)$.

From $\alpha \neq 1$ we infer the existence of an element $z$ in $G$ such that $z^{\alpha} \neq z$. Then there exists one and only one number $c \neq 0$ in $G$ such that

$$
\left(z^{\alpha}-z\right)^{\alpha}=c\left(z^{\alpha}-z\right) .
$$

If $t$ is any number in $G$, then it follows from the result, obtained in the preceding paragraph of this proof, that

$$
\left(t^{\alpha}-t\right)^{\alpha}=c\left(t^{\alpha}-t\right)
$$

is valid too. Apply this in particular on $t=z^{\alpha}$ and obtain $c\left(z^{\alpha^{2}}-z^{\alpha}\right)=\left(z^{\alpha^{2}}-z^{\alpha}\right)^{\alpha}=\left(\left(z^{\alpha}-z\right)^{\alpha}\right)^{\alpha}=\left(c\left(z^{\alpha}-z\right)\right)^{\alpha}=c^{\alpha}\left(z^{\alpha^{2}}-z^{\alpha}\right)$ proving $c=c^{\alpha}$, since $z \neq z^{\alpha}$ implies $z^{\alpha} \neq z^{\alpha^{2}}$, and this completes the proof of Lemma 4.

Theorem 4. Suppose that $\Pi$ is the projective plane over the field G, and that the projectivity $\phi$ of $\Pi$ is determined by the automorphism $\alpha$ of $G$ according to Lemma 3, (*). Then $\phi$ is a quasi-perspectivity, but not an involution ${ }^{12}$ if, and only if, there exists a number $c$ in $G$ which does not belong to the center of $G$ such that $c^{-1}+c$ is in the center of $G$ and such that

$$
x^{\alpha}=(1+c) x(1+c)^{-1} \text { for every } x \text { in } G .
$$

Proof. Assume firstly the existence of a number $c$ in $G$, not in the center of $G$, such that $c^{-1}+c$ is in the center of $G$ and such that

\footnotetext{
11 Note that $\alpha$ and $\phi$ are involutions if, and only if, $c=-1$.

${ }^{12}$ It is a consequence of Theorem 2 and Lemma 3 that $\phi$ cannot be a perspectivity
} either. 


$$
\begin{aligned}
x^{\alpha}=(1+c) x(1+c)^{-1} \text {. Then } c=c^{\alpha} \text { and } \\
\begin{aligned}
x^{\alpha^{2}}-c x c^{-1} & =(1+c)^{2} x(1+c)^{-2}-c x c^{-1} \\
& =\left[(1+c)^{2} x-c x c^{-1}(1+c)^{2}\right](1+c)^{-2} \\
& =\left[x+c^{2} x-c x\left(c^{-1}+c\right)\right](1+c)^{-2} \\
& =\left[x+c^{2} x-\left(c^{-1}+c\right) c x\right](1+c)^{-2}=0
\end{aligned}
\end{aligned}
$$

so that $x^{\alpha^{2}}=c x c^{-1}$. But $c$ is not in the center of $G$. Hence $\alpha^{2} \neq 1$, proving that $\phi$ is not an involution. Furthermore we find that

$$
\begin{aligned}
\left(x^{\alpha}-x\right)^{\alpha}-c\left(x^{\alpha}-x\right) & =x^{\alpha^{2}}-(1+c) x^{\alpha}+c x \\
& =x^{\alpha^{2}}-x^{\alpha^{2}}(1+c)+c x=-x^{\alpha^{2}} c+c x \\
& =-\left(c x c^{-1}\right) c+c x=0,
\end{aligned}
$$

and it follows from Lemma 4 that $\phi$ is a quasi-perspectivity. This proves the sufficiency of our condition.

Assume conversely that $\phi$ is a quasi-perspectivity, but not an involution. Then $\alpha^{2} \neq 1$, since $\phi^{2}$ is determined by $\alpha^{2}$, and we infer from Lemma 4 the existence of a number $c \neq 0$ in $G$ such that $c=c^{\alpha}$ and such that

$$
x^{\alpha^{2}}=(1+c) x^{\alpha}-c x \text { for every } x \text { in } G .
$$

From $\alpha^{2} \neq 1$ we infer $c \neq-1$.

If $y$ is an element in $G$, then we may apply (1) on $x=y^{2}$, and we find that

$$
\begin{aligned}
(1+c) y^{2 \alpha}-c y^{2}=y^{2 \alpha^{2}}=\left(y^{\alpha^{2}}\right)^{2} & =\left[(1+c) y^{\alpha}-c y\right]^{2} \\
= & (1+c) y^{\alpha}(1+c) y^{\alpha}-(1+c) y^{\alpha} c y-c y(1+c) y^{\alpha}+c y c y
\end{aligned}
$$

or

$$
(1+c) y^{\alpha}\left(y^{\alpha}-(1+c) y^{\alpha}+c y\right)=c y\left(y+c y-(1+c) y^{\alpha}\right) ;
$$

thus we have shown that

$$
(1+c) y^{\alpha} c\left(y-y^{\alpha}\right)=c y(1+c)\left(y-y^{\alpha}\right) \text { for every } y \text { in } G .
$$

If in particular $y \neq y^{\alpha}$, then we may divide by $y-y^{\alpha}$ in (2), proving that

$$
(1+c) y^{\alpha} c=c y(1+c) \text { for } y \neq y^{\alpha} .
$$

If $c$ were equal to 1 , then the characteristic of the field $G$ would be different from 2 , since we have already shown that $c \neq-1$. There exist elements $y \neq y^{\alpha}$, since $\alpha \neq 1$; we should infer from (3) that $y^{\alpha}=y$ 
is a consequence of $y \neq y^{\alpha}$ which is impossible. Thus we have shown that

$$
c \neq 0,1,-1 \text {. }
$$

If $w \neq w^{\alpha}$, then $y=w^{\alpha}-w$ is an element not equal to 0 , satisfying $y^{\alpha}=\left(w^{\alpha}-w\right)^{\alpha}=c\left(w^{\alpha}-w\right)=c y \neq y$ by (1) and (4). Hence we may apply (3) on this particular element $y$, and we obtain, because of (1),

$$
\begin{aligned}
c\left(w^{\alpha}-w\right)(1+c) & =c y(1+c)=(1+c) y^{\alpha} c=(1+c)\left(w^{\alpha}-w\right)^{\alpha} c \\
& =(1+c) c\left(w^{\alpha}-w\right) c .
\end{aligned}
$$

Since $c \neq 0$, this implies $\left(w^{\alpha}-w\right)(1+c)=(1+c)\left(w^{\alpha}-w\right) c$ or

$$
w^{\alpha}-w=c\left(w^{\alpha}-w\right) c \text { for every } w \text { in } G,
$$

since this formula (5) is trivially true, in case $w=w^{\alpha}$.

If $y \neq y^{\alpha}$, then we infer from (3) and (5) that

$$
\begin{aligned}
c y-y^{\alpha} c & =[c y(1+c)-c y c]-y^{\alpha} c=(1+c) y^{\alpha} c-c y c-y^{\alpha} c \\
& =c y^{\alpha} c-c y c=c\left(y^{\alpha}-y\right) c=y^{\alpha}-y ;
\end{aligned}
$$

and from this result we deduce that

$$
y^{\alpha}(1+c)=(1+c) y \text { whenever } y \neq y^{\alpha} .
$$

Consider now an element $x$ such that $x=x^{\alpha}$. We deduce from $\alpha \neq 1$ the existence of an element $z$ in $G$ such that $z \neq z^{\alpha}$. Then $y=x+z$ is an element in $G$, satisfying $y \neq y^{\alpha}$, and it follows from (6) that

$$
\begin{aligned}
x^{\alpha}(1+c) & =(y-z)^{\alpha}(1+c)=y^{\alpha}(1+c)-z^{\alpha}(1+c) \\
& =(1+c) y-(1+c) z=(1+c)(y-z)=(1+c) x .
\end{aligned}
$$

Combining this result with (6) and (4), we have shown that

$$
x^{\alpha}=(1+c) x(1+c)^{-1} \text { for every } x \text { in } G .
$$

If $x$ is an element in $G$, then it follows from (1) and (7) that

$$
\begin{aligned}
0 & =\left[x^{\alpha^{2}}-(1+c) x^{\alpha}+c x\right](1+c)^{2} \\
& =(1+c)^{2} x-(1+c)^{2} x(1+c)+c x(1+c)^{2} \\
& =c x(1+c)^{2}-(1+c)^{2} x c=c x-x c+c x c^{2}-c^{2} x c
\end{aligned}
$$

or, since $c \neq 0$ by (4),

$$
0=x c^{-1}-c^{-1} x+x c-c x
$$

or

$$
x\left(c+c^{-1}\right)=\left(c+c^{-1}\right) x \text { for every } x \text { in } G,
$$


proving that $c+c^{-1}$ is in the center of $G$. It follows from (7) and $\alpha \neq 1$ that $c$ cannot be in the center of $G$, and this completes the proof of the necessity of our condition.

CoROLlary 4. If $\Pi$ is the projective plane over the field $G$, then the following condition is necessary and sufficient for the existence of a quasiperspectivity which is neither a perspectivity nor an involution:

There exists a number $c$ in $G$ which does not belong to the center of $G$, though $c+c^{-1}$ is in the center.

This is an almost immediate consequence of Theorem 2, Lemma 3, and Theorem 4.

Corollary 5. If $\Pi$ is the projective plane over the commutative field $G$ then every quasi-perspectivity is a perspectivity or an involution, and the existence of quasi-perspectivities that are not perspectivities is equivalent to the existence of involutorial automorphisms not equal to 1 of $G$.

This is readily deduced from Corollary 4 , Theorem 2 and Lemma 3.

In order to show the existence of quasi-perspectivities that are neither perspectivities nor involutions we consider the projective plane II over the field $G$ of real quaternions. The number $i$ in $G$ satisfies $i^{2}=-i$ so that $i+i^{-1}=0$ is an element in the center of $G$, though $i$ itself is not in the center of $G$. Thus $i$ is an element $c$ meeting the requirements of Corollary 4, and this shows the existence of quasiperspectivities of $\Pi$ which are neither perspectivities nor involutions.

If $\Pi$ is the projective plane over the (not necessarily commutative) field $G$, and if $\Gamma$ is a projective subplane of $\Pi$, then $\Gamma$ is the projective plane over a suitable subfield $G_{0}$ of $G$. It is easy to establish a $1: 1$ and monotone increasing correspondence mapping the (partially ordered) set of the projective subplanes of $\Pi$ which contain $\Gamma$ upon the set of subfields of $G$ which contain $G_{0}$. In particular $\Gamma$ is maximal if, and only if, $G_{0}$ is maximal. Lemma 3 establishes a correspondence between the projectivities of $I I$ which leave invariant the elements in $\Gamma$ and the automorphisms of $G$ which leave invariant the elements in $G_{0}$. This shows that this type of Galois theory of projective planes may be reduced completely to the Galois theory of fields. If in particular $G$ is a commutative field, and if $G$ is a normal separable extension of $G_{0}$, then $G_{0}$ is maximal if, and only if, the degree of $G$ over $G_{0}$ is a prime $p$. In this case both the group of automorphisms of $G$ which leave invariant the elements in $G_{0}$ and the group $\mathrm{A}(\Gamma)$ of projectivities of $\Pi$ which leave invariant the elements in $\Gamma$ are of order $p$, and it is readily seen that the projectivities in $A(\Gamma)$ are quasi-perspectivities (involutions) if, and only if, $p=2$. This shows in particular that 
the maximality of $\Phi(\phi)$ does not imply that $\phi$ is a quasi-perspectivity.

We apply finally the results obtained so far to the special case of finite projective planes. If $\Pi$ is a finite projective plane, then every line carries the same number $n+1$ of points and every point is on $n+1$ lines; the total number of points (lines) is $1+n+n^{2}$. This integer $n$ $(\geqq 2)$ shall retain this significance throughout.

We denote by $N=N(\phi)$ the total number of fixed points ${ }^{13}$ of the projectivity $\phi$ of the finite projective plane $\Pi$.

Lemma 5. If $\phi$ is a projectivity of the finite projective plane $\Pi$, and if $n \leqq N$, then $\phi$ is a quasi-perspectivity.

Proof. Suppose that no fixed line passes through the point $W$. Since there exist at least two fixed points, there exists a fixed point $P \neq W$. If $W$ were a fixed point, then $P+W$ would be a fixed line which is impossible. Hence $W$ is not a fixed point.

Suppose next that there exists a fixed point $Z$ on the line $W+W \phi$. Then $Z$ is different from both $W$ and $W \phi$ so that

$$
(W+W \phi) \phi=(Z+W) \phi=Z+W \phi=W+W \phi .
$$

Thus the presence of a fixed point on $W+W \phi$ would imply that $W+W \phi$ is a fixed line through $W$. This is impossible. Hence there is no fixed point on the line $W+W \phi$.

If $P$ is any fixed point, then $W+P$ is not a fixed line. Thus it is impossible that $W+P$ carries more than one fixed point. But there exist at least $n$ fixed points each of which may be connected with $W$ by a line which does not carry any further fixed points. Since there pass exactly $n+1$ lines through $W$, it follows that the line $W+W \phi$ is the one and only line through $W$ which does not carry fixed points, and that all the other lines through $W$ carry one and only one fixed point.

Since no line through $W$ is a fixed line, no line through $W \phi$ is a fixed line. Hence it follows from the previous discussion that $W \phi+W \phi^{2}$ is the one and only one line through $W \phi$ which does not carry fixed points. But $W+W \phi$ is a fixed point free line passing through $W \phi$. Hence

$$
W+W \phi=W \phi+W \phi^{2}=(W+W \phi) \phi,
$$

proving that $W+W \phi$ is a fixed line passing through $W$. This is a contradiction which shows that every point is on a fixed line. It follows from Theorem 1 that $\phi$ is a quasi-perspectivity.

${ }^{13}$ It will be shown elsewhere that this is the number of fixed lines too. 
If $\phi$ is an elation, then the set of its fixed points is just the set of points on some suitable line, proving $N(\phi)=n+1$; if $\phi$ is a homology, ${ }^{14}$ then the set of its fixed points consists of all the points on its axis and of the center which is not on its axis so that $N(\phi)=n+2$.

THEOREM 5. The following properties of the projectivity $\phi$ of the finite projective plane II imply each other.

(i) $\phi$ is a quasi-perspectivity, but not a perspectivity.

(ii) The system $\Phi$ of fixed elements of $\phi$ is a projective subplane of $\Pi$ and $n=i^{2}$ where $i+1$ is the number of fixed points on a fixed line.

(iii) $n+2<N(\phi)$.

Proof. If (i) is satisfied by $\phi$, then we infer from Theorem 2 that $\Phi$ is a projective subplane of the finite plane $\Pi$. There exists therefore an integer $i \geqq 2$ such that every fixed line carries $i+1$ fixed points and such that $i+1$ fixed lines pass through any given fixed point. $N(\phi)=1+i+i^{2}$ is then the total number of fixed points and the total number of fixed lines. Consider a line $h$ which is not a fixed line. It is a consequence of Corollary 1 that $h$ carries one and only one fixed point $H$, and that every point $P \neq H$ on $h$ is situated on one and only one fixed line. Since every fixed line meets $h$ in one and only one point, and since exactly $i+1$ fixed lines pass through the fixed point $H$, it follows that the other $i^{2}$ fixed lines meet $h$ in the $n$ points different from $H$, proving that $n=i^{2}$. Thus we have shown that (ii) is a consequence of (i).

If (ii) is satisfied by $\phi$, then

$$
n+3=i^{2}+3 \leqq i^{2}+i+1=N(\phi)
$$

may be inferred from $2 \leqq i$, showing that (iii) is a consequence of (ii).

If finally (iii) is satisfied by $\phi$, then we infer from Lemma 5 that $\phi$ is a quasi-perspectivity. Since $N(\phi)=n+1$ or $n+2$ for perspectivities, $\phi$ cannot be a perspectivity, proving that (i) is a consequence of (iii).

CoRollary 6. Suppose that $\phi$ is a projectivity of the finite projective plane $\Pi$.

(a) $N(\phi) \neq n$.

(b) $n+1 \leqq N(\phi)$ if, and only if, $\phi$ is a quasi-perspectivity.

(c) $n+1=N(\phi)$ if, and only if, $\phi$ is an elation.

(d) $n+2=N(\phi)$ if, and only if, $\phi$ is a homology.

Proof. If $\phi$ is a quasi-perspectivity, then either $\phi$ is a perspectivity

${ }^{14}$ See Veblen-Young $[1$, p. 72]. 
and $N(\phi)=n+1$ or $n+2$, or else it follows from Theorem 5 that $n+2<N(\phi)$. If conversely $n+1 \leqq N(\phi)$, then it follows from Lemma 5 that $\phi$ is a quasi-perspectivity, proving (b). If furthermore $N(\phi)$ $=n+1$ or $n+2$, then it follows from Theorem 5 that $\phi$ is a perspectivity, and (c) and (d) are immediately inferred from a previous remark. If $n \leqq N(\phi)$, then it follows from Lemma 5 that $\phi$ is a quasi-perspectivity, and hence it follows from (b) that $n+1 \leqq N(\phi)$. Thus $n \leqq N(\phi)$ implies $n+1 \leqq N(\phi)$, proving (a).

\section{REINHOLD BAER}

\section{BIBLIOGRAPHY}

1. Homogeneity of projective planes, Amer. J. Math. vol. 64 (1942) pp. 137-152.

2. Null systems in projective spaces, Bull. Amer. Math. Soc. vol. 51 (1945) pp. 903906.

RICHARD BRAUER

1. A characterization of null systems in projective space, Bull. Amer. Math. Soc. vol. 42 (1936) pp. 247-254.

Marshall Hall

1. Projective planes, Trans. Amer. Math. Soc. vol. 54 (1943) pp. 229-277.

O. Veblen and J. W. Young

1. Projective geometry, vol. 1, Boston, 1910.

UNIVERSITY OF ILLINOIS 